\title{
Lyapunov function for nonuniform in time global asymptotic stability in probability with application to feedback stabilization.
}

\begin{abstract}
We extend the well-known Artstein-Sontag theorem by introducing the concept of control Lyapunov function for the notion of nonuniform in time global asymptotic stability in probability of stochastic differential system, when both the drift and diffusion terms are affine in the control. The main results of our work enable us to derive the necessary and sufficient conditions for feedback stabilization for affine in the control systems.
\end{abstract}

Keyword: Stochastic differential system; Control Lyapunov function; Global asymptotic stability in probability. 